\title{
The dipeptidyl peptidase-4 inhibitor sitagliptin suppresses mouse colon tumorigenesis in type 2 diabetic mice
}

\author{
NAOKI YORIFUJI, TAKUYA INOUE, MUNETAKA IGUCHI, KAORI FUJIWARA, KAZUKI KAKIMOTO, \\ SADAHARU NOUDA, TOSHIHIKO OKADA, KEN KAWAKAMI, YOSUKE ABE, \\ TOSHIHISA TAKEUCHI and KAZUHIDE HIGUCHI
}

Second Department of Internal Medicine, Osaka Medical College, Takatsuki City, Osaka 569-8686, Japan

Received August 27, 2015; Accepted October 14, 2015

DOI: $10.3892 /$ or.2015.4429

\begin{abstract}
Patients with type 2 diabetes mellitus are known to have an increased risk of colorectal neoplasia. Dipeptidyl peptidase-4 (DPP-4) inhibitors have been used as a new therapeutic tool for type 2 diabetes. Since the substrates for DPP-4 include intestinotrophic hormones and chemokines such as GLP-2 and stromal cell-derived factor-1 (SDF-1), which are associated with tumor progression, DPP-4 inhibitors may increase the risk of colorectal tumors. However, the influence of DPP-4 inhibitors on colorectal neoplasia in patients with type 2 diabetes remains unknown. In the present study, we show that long-term administration of a DPP-4 inhibitor, sitagliptin (STG), suppressed colon carcinogenesis in leptin-deficient (ob/ob) C57BL/6J mice. Colonic mucosal concentrations of glucagon-like peptide-1 (GLP-1) and GLP-2 were significantly elevated in the ob/ob mice. However, mucosal GLP concentrations and the plasma level of SDF-1 were not affected by the administration of STG. Real-time PCR analysis revealed that colonic mucosal IL-6 mRNA expression, which was significantly upregulated in the ob/ob mice, was significantly suppressed by the long-term administration of STG. These results suggest that a DPP-4 inhibitor may suppress colon carcinogenesis in mice with type 2 diabetes in a GLP-independent manner. Since DPP-4 has multiple biological functions, further studies analyzing other factors related to colon carcinogenesis are needed.
\end{abstract}

\section{Introduction}

In recent years, the number of the patients with type 2 diabetes has increased in Japan since Japanese people have experienced a rapid and drastic change in life style, including

Correspondence to: Dr Takuya Inoue, Second Department of Internal Medicine, Osaka Medical College, 2-7 Daigakumachi, Takatsuki City, Osaka 569-8686, Japan

E-mail: ureuretakuwan@yahoo.co.jp

Key words: diabetes, dipeptidyl peptidase, colorectal neoplasia, glucagon-like peptide-2 dietary changes and an increased rate of obesity, which is the primary risk factor for type 2 diabetes $(1,2)$. Paralleling these changes, rates of cancers of the colon, pancreas, breast and prostate, which are known to be more common in Western countries, have increased in Japan (2-4). Among these cancers, epidemiological studies have shown that obesity increases the risk of colon cancer by 1.5-2.0-fold with obesity-associated colon cancer accounting for $14-35 \%$ of total incidence (5). Patients with type 2 diabetes mellitus are also known to have an increased risk of colorectal neoplasia compared with those without diabetes (6).

The gut incretin hormone glucagon-like peptide-1 (GLP-1) is released from enteroendocrine cells in response to ingested nutrients and acts as a key determinant of blood glucose homeostasis by virtue of its ability to slow gastric emptying, to enhance pancreatic insulin secretion, and to suppress pancreatic glucagon secretion (7-9). Since GLP-1 is rapidly degraded and deactivated by dipeptidyl peptidase-4 (DPP-4), DPP-4 inhibitors, such as sitagliptin (STG), vildagliptin and saxagliptin, have recently emerged as therapeutic options for the treatment of type 2 diabetes $(10,11)$.

Another processing product of proglucagon, GLP-2, is also a DPP-4 substrate. GLP-2 is a pleiotropic hormone that affects multiple facets of intestinal physiology, including epithelial growth, barrier function, digestion, absorption, motility and blood flow $(12,13)$. GLP-2 exerts potent anti-apoptotic effects in the gastrointestinal tract, so GLP-2 is a promising target for the development of novel treatments for intestinal diseases. Since DPP-4 rapidly degrades and deactivates GLP-2, DPP-4 inhibitors are also considered novel approaches to the treatment of intestinal inflammation $(14,15)$. Indeed, we recently reported a protective effect of DPP-4 inhibitors on indomethacin-induced intestinal injury in rats $(16,17)$. However, in regard to colon carcinogenesis, exogenous and endogenous GLP-2 has been reported to enhance colon carcinogenesis in azoxymethanetreated mice (18). Moreover, since the substrates for DPP-4 include growth factors and chemokines, such as insulin-like growth factor-1 (IGF-1) and stromal cell-derived factor-1 (SDF-1), which are associated with tumor progression, DPP-4 inhibitors may increase the risk of colorectal tumors (19). However, the influence of DPP-4 inhibitors on colorectal neoplasia in patients with type 2 diabetes is unknown. Therefore, in the present study, we evaluated the effect of a 
DPP-4 inhibitor, STG, on diabetes-related mouse colon carcinogenesis and proteomic changes.

\section{Materials and methods}

Animals. Six-week-old male wild-type (WT) and leptin-deficient (ob/ob) C57BL/6J mice (Oriental Bio Service, Inc., Kyoto, Japan) were used in the present study. The animals were maintained in an animal colony with controlled temperature $\left(23^{\circ} \mathrm{C}\right)$ and light (12/12-h light and dark cycle) at the Osaka Medical College (OMC), Osaka, Japan and were permitted free access to standard mouse chow pellets (MM-3; Funabashi, Chiba, Japan) and tap water. Ethical permission for the experiments presented in the present study was given by the Animal Ethics Committee of the Osaka Medical College and all procedures were conducted according to the guidelines of the Institute for Laboratory Animal Research at Osaka Medical College.

Protocol for induction of colorectal tumors and experimental procedures. Colorectal tumors and experimental colitis were induced as previously described (20). In brief, the mice received 1,2-dimethylhydrazine (DMH) (Wako Pure Chemical Industries, Osaka, Japan) subcutaneously three times per week at a dose of $20 \mathrm{mg} / \mathrm{kg}$ body weight. Starting 1 week after DMH injection, chronic colitis was induced by the administration of 2 cycles of dextran sulfate sodium (DSS) (molecular weight, 4,000-5,000; Meitou Sangyou, Osaka, Japan; each cycle consisted of $3 \%$ DSS for 7 days and then distilled water for 14 days). The mice were sacrificed 28 days after completion of the 2 cycles. The following groups were used in the experiment: WT, ob/ob and ob/ob+STG groups. We previously evaluated the effect of STG $(0.3-3 \mathrm{mg} / \mathrm{kg}$, i.g.) on mucosal DPP activity and reported that oral administration of STG dose-dependently suppressed mucosal DPP activity (16). Therefore, in the present study, the DPP-4 inhibitor sitagliptin phosphate monohydrate (Carbosynth Limited, Berkshire, UK) $(3 \mathrm{mg} / \mathrm{kg})$, was given to mice by oral gavage every day during the experimental period.

The entire colorectum from the colocecal junction to the anal verge was excised and rinsed in phosphate-buffered saline (PBS). The specimen was longitudinally opened and was fixed on a cork board in $10 \%$ formalin. Then, the specimen was stained with $0.2 \%$ methylene blue, and the number of colonic tumors and aberrant crypt foci (ACF) was counted under a stereomicroscope.

Mucosal DPP-4 activity. Mucosal scraped tissues were collected in lysis buffer (TRIS-buffered saline containing protease inhibitors) (Complete Mini; Roche Diagnostics, Indianapolis, IN, USA) with or without the DPP-4 inhibitor, K-579 (Tocris Bioscience, Ellisville, MO, USA). Blood samples were also collected into chilled Eppendorf tubes containing $1 \mu \mathrm{l}$ each of $0.5 \mathrm{M}$ EDTA and $100 \mu \mathrm{M} \mathrm{K}-579$. The samples were immediately centrifuged at 3,000 $\mathrm{x}$ g for $5 \mathrm{~min}$ and their supernatants and plasma were stored at $-80^{\circ} \mathrm{C}$ until measurement. Mucosal DPP-4 activity was determined by the cleavage rate of 7-amino-4-methylcoumarin (AMC; SensoLyte AMC DPPIV Assay kit; AnaSpec, Inc., Fremont, CA, USA) from the synthetic substrate according to the manufacturer's instructions. In brief, each sample of scraped colon was homogenized.
Table I. Primers used to detect mRNA expression levels.

\begin{tabular}{lll}
\hline Genes & \multicolumn{2}{c}{ Sequence of primers } \\
\hline TNF- $\alpha$ & S & 5'-TATGGCCCAGACCCTCACA-3' \\
& A & 5'-GGAGTAGACAAGGTACAACCCATC-3' \\
IGF-1 & S & 5'-ACTGCTAAACACTTGGCAGGAG-3' \\
& A & 5'-TTGCAAGGTTTAAGGATACAGAGAC-3' \\
IL-6 & S & 5'-CCACTTCACAAGTCGGAGGCTTA-3' \\
& A & 5'-CCAGTTTGGTAGCATCCATCATTTC-3' \\
\multirow{3}{*}{-actin } & S & 5'-CATCCGTAAAGACCTCTATGCCAAC-3' \\
& A & 5'-ATGGAGCCACCGATCCACA-3' \\
\hline
\end{tabular}

$\mathrm{S}$, sense; A antisense; TNF- $\alpha$, tumor necrosis factor- $\alpha$; IGF-1, insulin-like growth factor-1; IL-6, interleukin-6.

Lysates containing $10 \mu \mathrm{g} / 50 \mu \mathrm{l}$ of protein were mixed with $50 \mu \mathrm{l}$ of the DPP-4 substrate AMC. After 30 min of incubation at room temperature, AMC release was determined using a fluorescence plate reader (excitation at $354 \mathrm{~nm}$ and emission at $442 \mathrm{~nm}$ ) (Fluoroskan Ascent; Thermo Fisher Scientific K.K., Kanagawa, Japan). DPP activity in mucosa was measured as the relative fluorescence units (RFUs), and then calibrated using the concentration of Gly-Pro AMC $(\mu \mathrm{M})$ as a reference.

Measurement of plasma and mucosal GLPS. Plasma and mucosal active GLP-1 (7-36) and total GLP-2 were measured using a GLP-1 (active 7-36) ELISA kit and GLP-2 (mouse) ELISA kit (ALPCO Diagnostics, Salem, NH, USA) according to the manufacturer's instructions.

Analysis of adipokine-related proteins. Mouse adipokine array kits (Proteome Profiler Array and Human Cytokine; R\&D Systems, Minneapolis, MN, USA) were used to simultaneously detect the relative expression levels of 38 different obesity-related proteins. The array was performed according to the manufacturer's instructions. Blots were developed using enhanced chemiluminescence and a Fuji Film Imaging System (Application Note LAS-3000; Fuji Film, Tokyo, Japan).

Real-time quantitative polymerase chain reaction. To measure mRNA expression of the pro-inflammatory cytokines interleukin (IL)-6, tumor necrosis factor (TNF)- $\alpha$ and IGF-1, a small amount of colonic mucosa was scraped with a glass slide, frozen in liquid nitrogen and stored at $-80^{\circ} \mathrm{C}$ until RNA isolation. Total RNA was extracted using the total RNeasy mini kit (Qiagen, Tokyo, Japan). For cDNA synthesis, RNA was reverse-transcribed with a PrimeScript RT reagent kit with the SYBR Premix Ex Taq kit (both from Takara Bio Inc., Shiga, Japan) on a Thermal Cycler Dice real-time system TP870 (Takara Bio Inc.). The sequences of the sense and antisense primers for mouse IL- 6 , TNF- $\alpha$, IGF- 1 and $\beta$-actin are shown in Table I. Cycling conditions were one cycle of $95^{\circ} \mathrm{C}$ for $30 \mathrm{sec}$ for denaturing followed by 40 cycles of $95^{\circ} \mathrm{C}$ for $5 \mathrm{sec}$, and $60^{\circ} \mathrm{C}$ for $30 \mathrm{sec}$, and one cycle of $95^{\circ} \mathrm{C}$ for $15 \mathrm{sec}$, and $60^{\circ} \mathrm{C}$ for $30 \mathrm{sec}$. The expression of target genes was normalized to the expression of $\beta$-actin. 


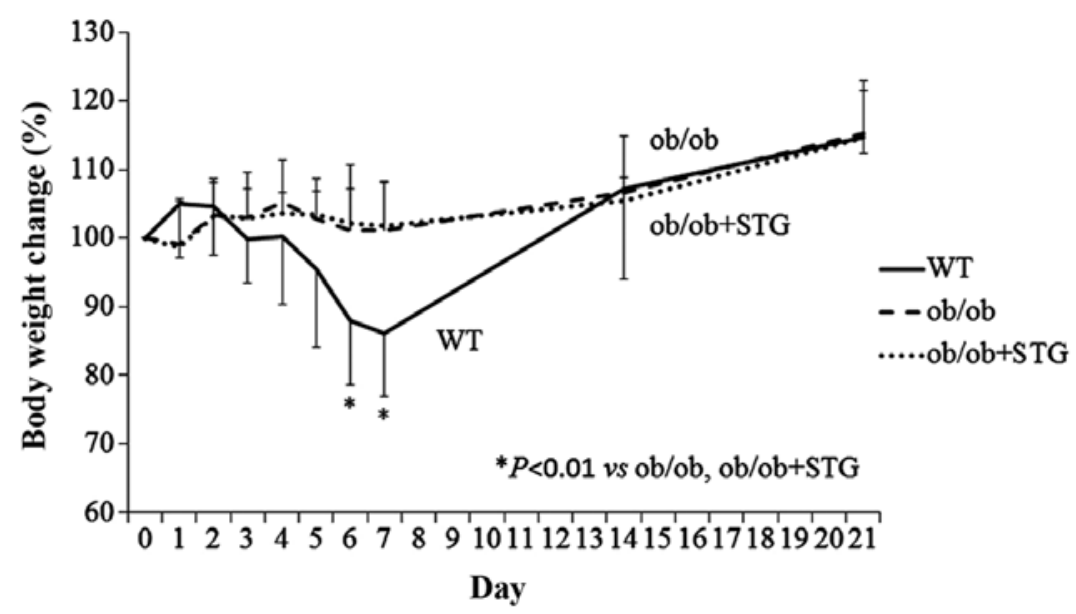

Figure 1. Time course of body weight change (\%) during the first course of DSS administration (3\% DSS for 7 days followed by distilled water for 14 days). Body weight change (\%) was calculated for each mouse by dividing its body weight on the specified day by body weight at day 0 . * $\mathrm{P}<0.01 \mathrm{vs.} \mathrm{ob/ob,} \mathrm{ob/ob+STG.}$

Statistical analysis. The data are expressed as means \pm SD. Data were derived from six animals in each group. Comparisons were performed using one-way ANOVA or Kruskal-Wallis followed by the Fisher's PLSD test. Statistical significance was defined as $\mathrm{P}<0.05$.

\section{Results}

Effects of chronic administration of STG on body weight, mortality and number of colorectal tumors. In most of the C57BL/6J mice that received 3\% DSS, body weight loss was observed 3 days after DSS administration (Fig. 1). Clinical symptoms of colitis, including loose stools and loss of body weight, progressed during DSS treatment. These symptoms gradually disappeared during the period of drinking distilled water without DSS for the following 14 days. Percent body weight change was significantly smaller in ob/ob mice than in the WT mice at 6 and 7 days after DSS treatment. Body weight was significantly increased in ob/ob and ob/ob+STG mice. Administration of STG did not significantly change body weight in ob/ob mice during DSS treatment $(21.5 \pm 0.4$, $51.2 \pm 2.7$ and $52.5 \pm 1.1 \mathrm{~g}$ in the WT, ob/ob and ob/ob+STG groups, respectively). Plasma glucose measured before sacrifice was also not affected by the administration of STG in ob/ob mice $(225.5 \pm 21.1,251.9 \pm 5.3$ and $264.7 \pm 8.4 \mathrm{mg} / \mathrm{dl}$ in the WT, ob/ob and ob/ob+STG groups, respectively). However, administration of STG did significantly improve survival of ob/ob mice over the entire experimental period (Fig. 2). All C57BL/6J mice that received DMH pretreatment and repeated administration of DSS developed multiple colorectal tumors (Fig. 3). The mean number of tumors/ACF was $7.8 \pm 4.5 / 6.1 \pm 4.7,10.6 \pm 3.3 / 12.4 \pm 6.2$ and $6.7 \pm 2.4 / 3.1 \pm 2.4$ in the WT, ob/ob and ob/ob+STG groups, respectively (Fig. 4). Administration of STG significantly lowered the number of $\mathrm{ACF}$ and tumors+ACF in ob/ob mice.

Mucosal DPP activity and the concentration of plasma and mucosal GLPs. Since it has been reported that endogenous GLP-2 enhances colon carcinogenesis (18), we evaluated the effect of STG on mucosal DPP activity and measured the

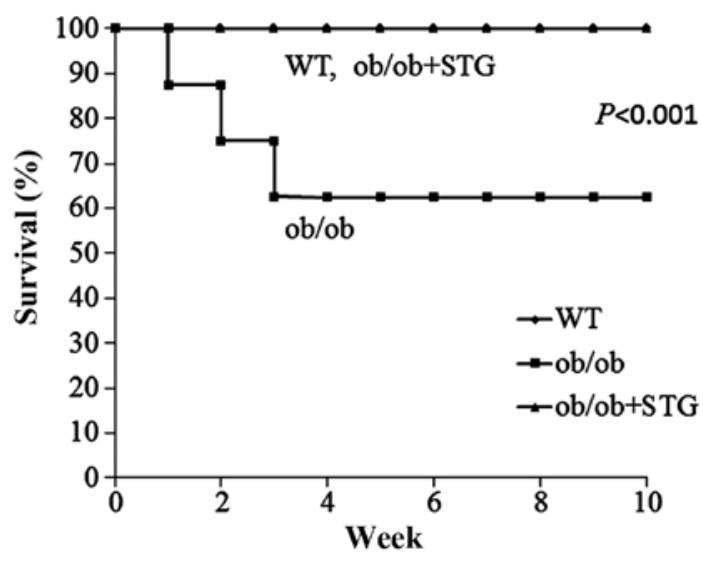

Figure 2. Survival rate over the entire experimental period was compared among WT, ob/ob and ob/ob+STG mice. Administration of STG significantly improved the survival rate of ob/ob mice $(\mathrm{P}<0.001)$.

concentrations of plasma and mucosal GLPs. As we previously reported, the administration of STG did not significantly suppress mucosal DPP activity in the colon (Fig. 5) (16). Accordingly, the mucosal concentration of active GLP-1 was not significantly elevated by STG administration, while the concentrations of mucosal total GLP-1 and GLP-2 were significantly increased in ob/ob mice compared to those in WT mice. Plasma concentration of total GLP-2 was also elevated in ob/ob mice. However, the administration of STG did not affect the plasma concentration of total GLP-1 or GLP-2 (Fig. 5).

Analysis of adipokine-related proteins. During the experimental period, the administration of STG did not significantly affect body weight in ob/ob mice. Plasma and mucosal GLPs were significantly elevated in ob/ob mice compared to WT mice. However, the effects of STG on the plasma and mucosal GLP concentrations in ob/ob mice were minimal. Therefore, a protein array was performed to examine changes in the expression of various adipokines and adipokine-related proteins (Fig. 6). The protein array showed an elevated plasma 
A

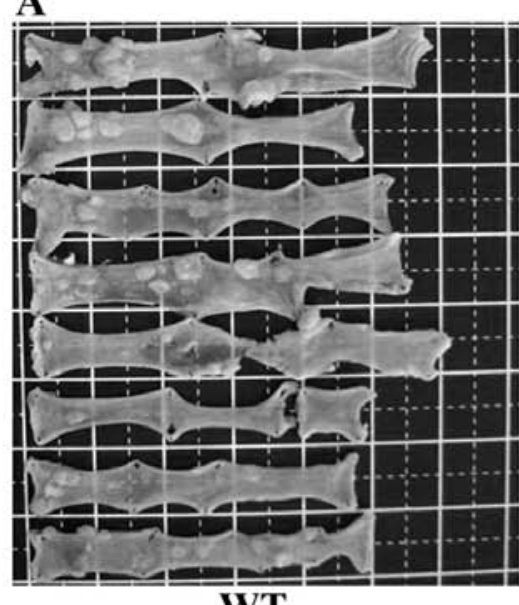

WT
B

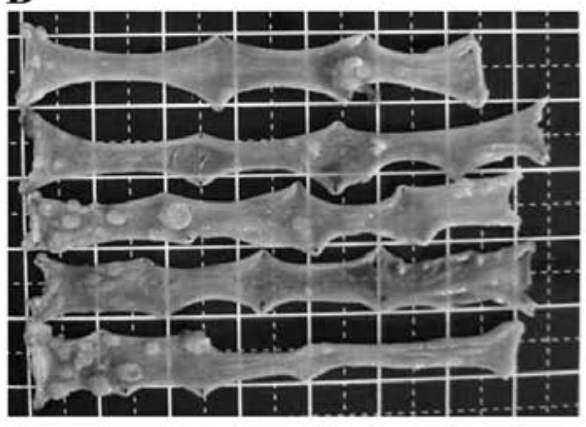

C

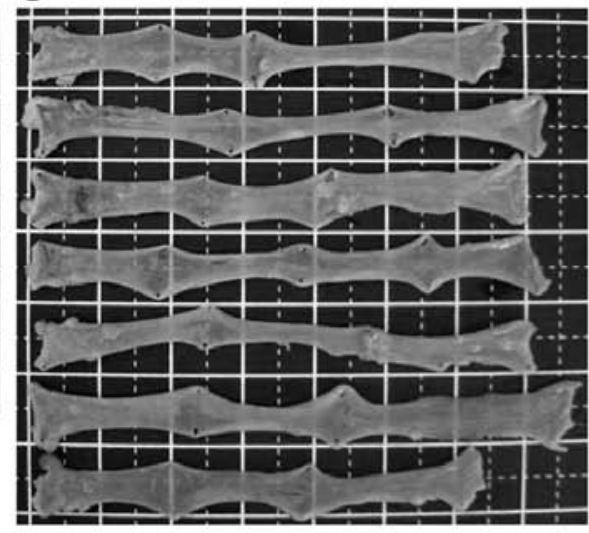

ob/ob+STG

Figure 3. Colorectal tumors in mice. Macroscopic view showing gross lesions in the colon. (A) WT; (B) ob/ob; (C) ob/ob+STG.

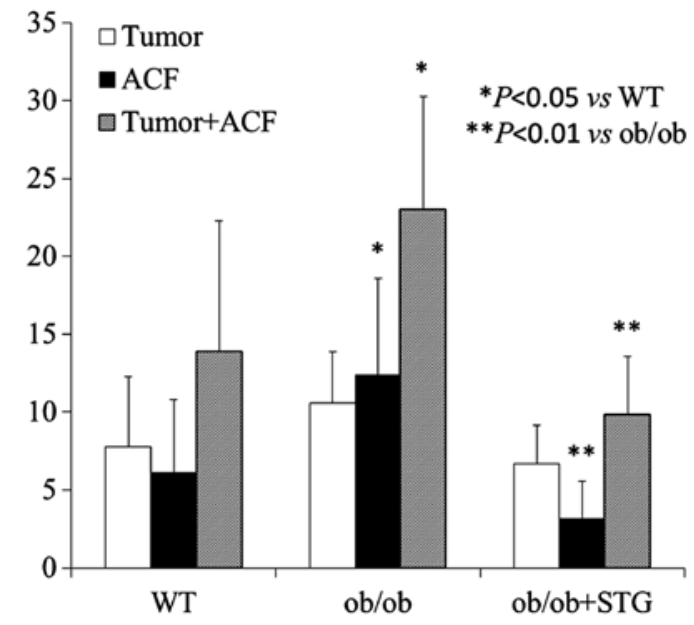

Figure 4. The number of DMH/DSS-induced tumors and ACF. In ob/ob mice, the total number of tumors and ACF was significantly increased compared to those of WT mice. Administration of STG significantly suppressed the number of tumors and ACF in ob/ob mice. ${ }^{*} \mathrm{P}<0.05$ vs. WT, ${ }^{* *} \mathrm{P}<0.01 \mathrm{vs}$. ob/ob.

level of resistin in the ob/ob group and a decreased plasma level of IGF-binding proteins (IGFBPs) in the ob/ob+STG group. The administration of STG did not significantly decrease the plasma concentration of resistin, suggesting that chronic administration of STG does not significantly alter metabolic status in ob/ob mice.

Mucosal TNF- $\alpha, I L-6$ and IGF-1 mRNA expression in the colon. It is well known that TNF- $\alpha$, IL-6 and IGF-1 influence all stages of tumor development, including initiation, promotion and development. To analyze the effects of topical STG on mucosal TNF- $\alpha$, IL-6 and IGF-1 in ob/ob mouse colon, we examined mucosal TNF- $\alpha$, IL-6, and IGF-1 mRNA expression in the colon (Fig. 7). Real-time PCR analysis revealed that colonic mucosal IL-6 mRNA expression was significantly upregulated in ob/ob mice compared to WT mice and that the administration of STG significantly suppressed this upregulation, while mucosal expression of TNF- $\alpha$ and IGF-1 was not affected.

\section{Discussion}

In the present study, we demonstrated that long-term administration of a DPP-4 inhibitor, sitagliptin (STG), showed protective effects against colorectal neoplasia in type 2 diabetic mice. It is well known that patients with type 2 diabetes have an increased risk of colorectal neoplasia compared with those without diabetes, and in recent years, DPP-4 inhibitors, which stabilize incretin hormones, have been widely used to treat patients with diabetes. DPP-4 inhibitors may stabilize other hormones and cytokines that are associated with colon tumorigenesis. Despite this observation, to date there have been very few studies investigating the relationship between DPP-4 inhibitors and colon carcinogenesis (21).

Generally, patients with type 2 diabetes have an increased risk of cardiovascular disease and many take NSAIDs, such as low-dose aspirin, to reduce the risk of cardiovascular and cerebrovascular thrombosis $(22,23)$. However, NSAIDs can induce gastroenteropathy by increasing the permeability of the intestinal mucosa $(24,25)$. Since the intestinotrophic hormone GLP-2 is rapidly degraded and deactivated by DPP-4, we previously evaluated the effect of DPP-4 inhibitor on non-steroidal anti-inflammatory drug (NSAID)-induced enteritis in mice and reported that STG has a preventive effect against enteric ulcers and promotes ulcer healing via activation of the GLP-2 pathway $(16,17)$. However, since chronic treatment with GLP-2 enhances colon carcinogenesis in azoxymethan-treated mice and since antagonism of the GLP-2 receptor decreases dysplasia, exogenous and endogenous GLP-2 has been suspected to promote colon carcinogenesis in mice $(18,26)$. Moreover, DPP-4 cleaves and deactivates other hormones and cytokines which are associated with tumor progression, such as SDF-1 and IGF-1 (19). Femia et al investigated whether long-term administration of STG affects colon carcinogenesis in rats and showed that chronic STG administration in DMH-induced rats reduced the number of precancerous lesions, mucin-depleted foci, and blood reactive oxygen species, suggesting a protective effect against carcinogenesis. However, the effect of STG on the number of colorectal tumors and levels of substrates related to colon 


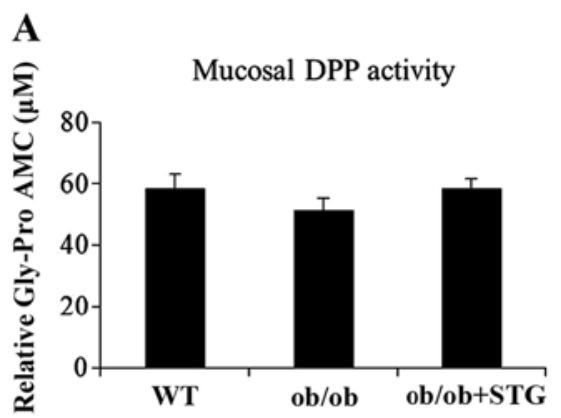

D Mucosal active GLP-1

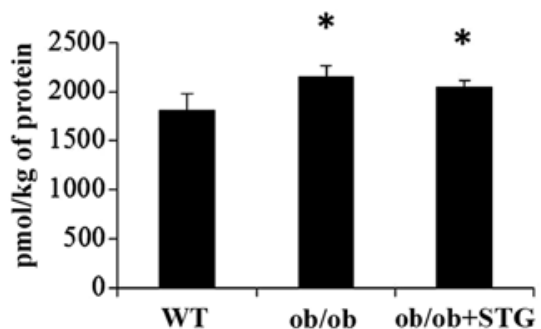

B

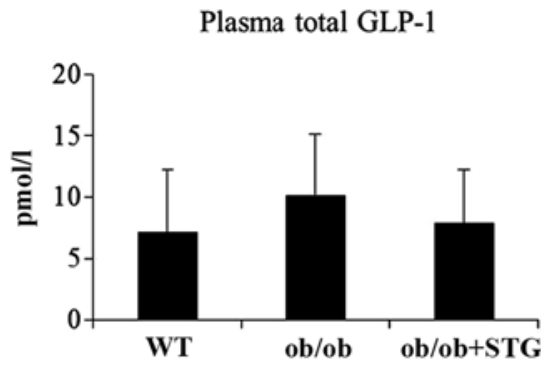

$\mathbf{E}$

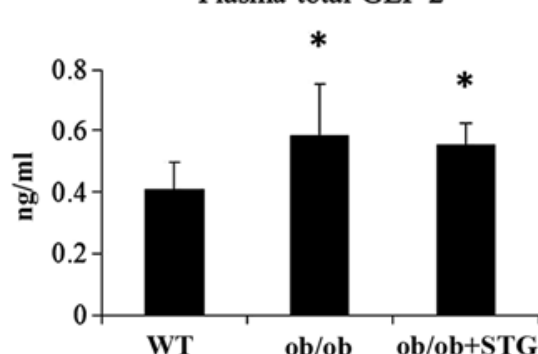

C

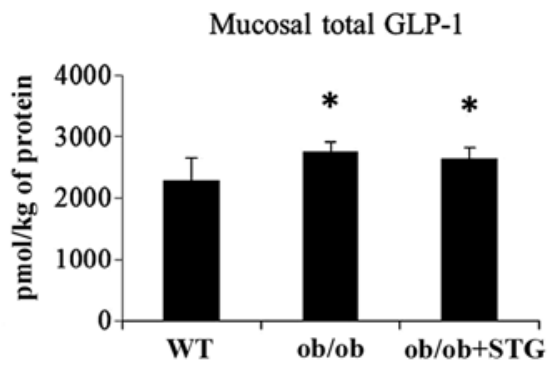

$\mathbf{F}$

Mucosal total GLP-2

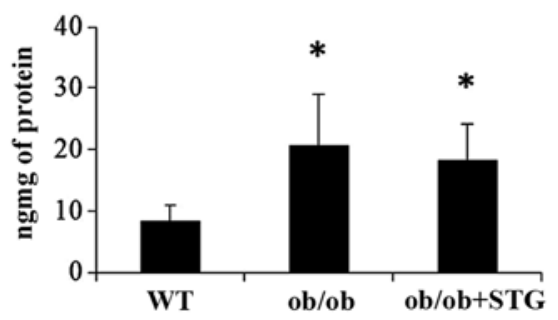

${ }^{*} P<0.05$ vs WT

Figure 5. (A) Mucosal DPP enzyme activity, (B and C) plasma and mucosal concentrations of total GLP-1 and (E and F) GLP-2 and mucosal concentration of active GLP-1 (D). DPP enzymatic activity was measured as relative RFU and then calibrated and expressed using the Gly-Pro AMC ( $\mu$ M) reference. The administration of STG did not significantly affect DPP enzymatic activity or mucosal GLP concentrations in the colon. ${ }^{*} \mathrm{P}<0.05$ vs. WT.

$\mathbf{A}$

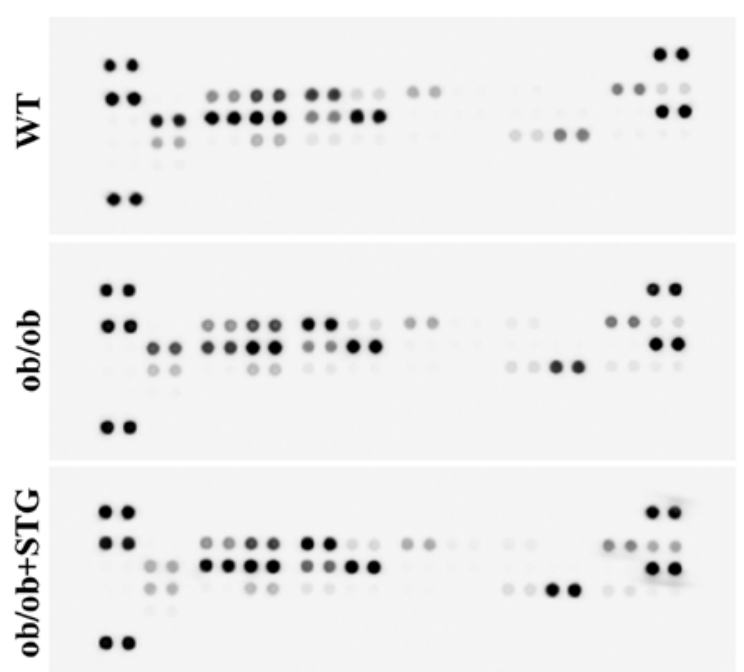

B

\begin{tabular}{|c|c|c|c|c|c|c|c|c|c|c|c|c|c|c|c|}
\hline & 1 & 3 & 6 & 7 & 10 & 11 & 14 & 15 & 16 & 17 & 18 & 19 & 20 & 21 & 23 \\
\hline $\mathbf{A}$ & PC & & & & & & & & & & & & & & PC \\
\hline B & Adiponectin & AbRP & ANGPT-L3 & CRP & DPP-4 & Endocan & Fetvin A & FGF & acidic & & $8-21$ & & & ICAM-1 & IGF-I \\
\hline $\mathrm{C}$ & IGF-II & IGFBP-1 & IGFBP-2 & IGFBP-3 & IGFBP-5 & IGFBP-6 & IIL-6 & II & -10 & & -11 & Le & & LIF & Lipocalin-2 \\
\hline D & MCP-1 & M-CSF & Oncostatin M & Pentexin 2 & Pentaxin 3 & Pref-1 & RAGE & RAN & VTES & & $3 \mathrm{P} 4$ & Ret & istin & Serpin El & TIMP-1 \\
\hline E & $\mathrm{TNF}-\alpha$ & VEGF & & & & & & & & & & & & & \\
\hline $\mathbf{F}$ & PC & & & & & & & & & & & & & & NC \\
\hline
\end{tabular}

Figure 6. The mouse adipokine and adipokine-related protein array in plasma. A Proteome Profiler Mouse Adipokine Array kit (R\&D Systems) containing 38 mouse adipokine and adipokine-related antibodies in duplicate was used to detect adipokines in the plasma (A). A position map (B).

carcinogenesis has not been previously evaluated. Therefore, we sought to clarify the long-term effects of a DPP-4 inhibitor on colon carcinogenesis. We found no significant difference in plasma and mucosal GLP expression or plasma concentra- 
A

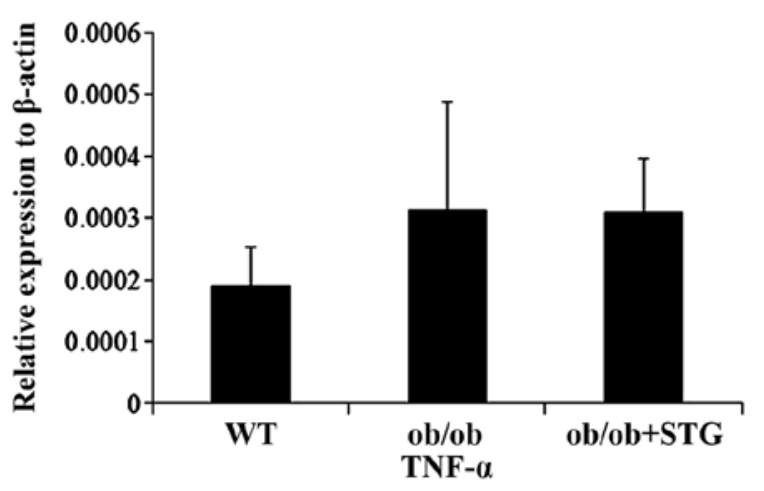

C

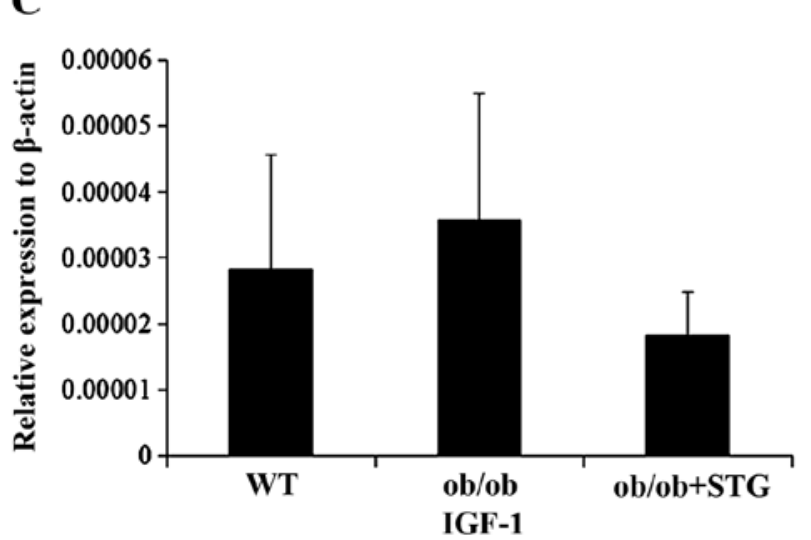

$\mathbf{B}$

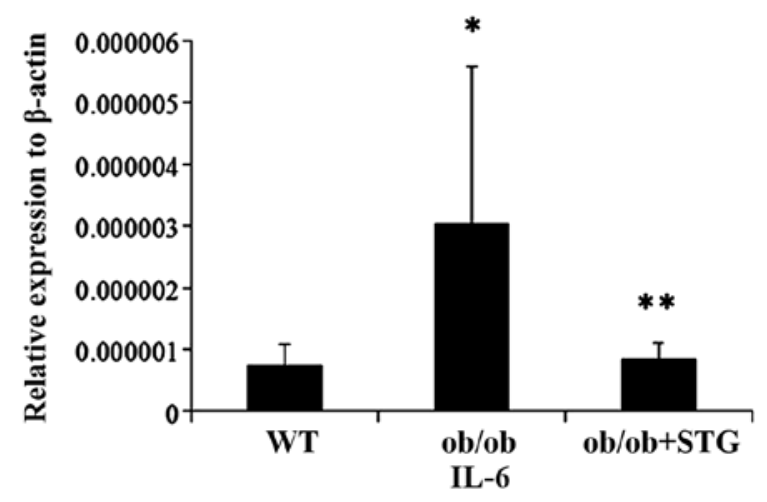

${ }^{*} P<0.05$ vs WT

${ }^{* *} P<0.05$ vs ob/ob

Figure 7. Mucosal mRNA expression of TNF- $\alpha$, IL-6 and IGF-1 in the colon. mRNA expression of TNF- $\alpha$ (A), IL-6 (B) and IGF-1 (C) was measured and expressed relative to $\beta$-actin. ${ }^{*} \mathrm{P}<0.05$ vs. WT, ${ }^{* *} \mathrm{P}<0.05$ vs. ob/ob.

tions of SDF-1 (data not shown) between ob/ob mice and ob/ob mice treated with long-term STG administration. As we previously reported, the oral administration of STG dosedependently suppresses mucosal DPP activity and increases mucosal active GLP concentration in the small intestine but not in the colon since DPP4 mRNA is mainly expressed in the small intestine and DPP activity is highly correlated to DPP4 mRNA expression (16). DPP-4 shares sequence homology with the enzymes DPP-8, DPP-9 and fibroblast activation protein. Since DPP-8 and DPP-9 are mainly expressed in the colon and STG selectively inhibits DPP-4 activity, we consider that the effect of DPP-4 inhibitor may be minimal in the colonic mucosa (27). Regarding systemic effect, STG also showed minimal effect on metabolic status since obese mutant mice ate excessively during the entire period of the experiment. Generally, DPP4 inhibitor may regulate appetite and body weight. Actually, we previously conducted the experiment to evaluate the effect of STG on high-fat dieted mice. In that study, the administration of STG significantly affected adipokines (28). In the present study, since we used obese mutant mice (ob/ob mice), they always ate excessively during the whole period of the experiment although they received STG. However, the administration of STG showed the suppressive effect of colon tumorigenesis. These results suggest that DPP4 inhibitor may suppress colon tumorigenesis in a GLP-independent manner.

In obese patients, several factors, including increased levels of blood insulin, IGF-1, leptin, TNF- $\alpha$, IL-6 and decreased adiponectin, are altered and are considered to promote cancer development (5). Therefore, we next conducted proteomic analysis using an adipokine array and found that plasma levels of adiponectin, IGF-1, TNF- $\alpha$ and IL- 6 were not affected by administration of STG. These findings suggest that long-term administration of STG may have minimal effects on the metabolic status of ob/ob mice. Rather, as previously reported, administration of STG decreased the plasma concentration of IGF-BP, suggesting IGF-1 signaling is upregulated by this DPP-4 inhibitor (29). Taken together, these results suggest that long-term administration of STG suppresses colon carcinogenesis in ob/ob mice without altering metabolic status, including insulin resistance. Notably, real-time PCR analysis revealed that mucosal expression of IL-6 mRNA was significantly upregulated in ob/ob mice compared to WT mice and was suppressed by long-term administration of STG. Insulin has been known to increase IL-6, which is a key regulator of colorectal cancer gene expression through activation of cGMP. In the present study, although plasma and colonic mucosal DPP activity was not affected by long-term administration of STG, topical (colonic mucosal) expression of IL-6 was significantly suppressed. Since some studies demonstrated that DPP-4 enhanced binding of transcription factors to the IL-6 promoters and increased IL-6 protein expression and that DPP-4 inhibition suppressed IL-6 production, we believe that the oral administration of STG suppresses colonic mucosal IL-6 independent of mucosal DPP activity and mucosal GLP concentration $(27,30,31)$. 
In conclusion, long-term oral administration of the DPP-4 inhibitor STG, suppressed mucosal IL-6 expression and colon carcinogenesis in a mouse model of type 2 diabetes, although metabolic abnormalities were not improved. These effects may be independent of mucosal DPP activity and mucosal GLP levels. Although further studies are needed to clarify the mechanisms by which this DPP-4 inhibitor suppresses colon carcinogenesis, DPP-4 inhibitors may be very useful for patients with type 2 diabetes, who have an increased risk of colorectal carcinogenesis.

\section{References}

1. Tsugane $\mathrm{S}$ and Inoue $\mathrm{M}$ : Insulin resistance and cancer: Epidemiological evidence. Cancer Sci 101: 1073-1079, 2010.

2. Neville SE, Boye KS, Montgomery WS, Iwamoto K, Okamura M and Hayes RP: Diabetes in Japan: A review of disease burden and approaches to treatment. Diabetes Metab Res Rev 25: 705-716, 2009.

3. Siegel R, Naishadham D and Jemal A: Cancer statistics, 2013. CA Cancer J Clin 63: 11-30, 2013.

4. Katanoda K, Matsuda T, Matsuda A, Shibata A, Nishino Y, Fujita M, Soda M, Ioka A, Sobue T and Nishimoto H: An updated report of the trends in cancer incidence and mortality in Japan. Jpn J Clin Oncol 43: 492-507, 2013.

5. Huang XF and Chen JZ: Obesity, the PI3K/Akt signal pathway and colon cancer. Obes Rev 10: 610-616, 2009.

6. Berster JM and Göke B: Type 2 diabetes mellitus as risk factor for colorectal cancer. Arch Physiol Biochem 114: 84-98, 2008.

7. Reimer MK, Holst JJ and Ahrén B: Long-term inhibition of dipeptidyl peptidase IV improves glucose tolerance and preserves islet function in mice. Eur J Endocrinol 146: 717-727, 2002.

8. Nadkarni P, Chepurny OG and Holz GG: Regulation of glucose homeostasis by GLP-1. Prog Mol Biol Transl Sci 121: 23-65, 2014.

9. Frezza EE, Wachtel MS and Chiriva-Internati M: The multiple faces of glucagon-like peptide-1 - obesity, appetite, and stress: What is next? A review. Dig Dis Sci 52: 643-649, 2007.

10. Takasaki K, Takada H, Nakajima T, Ueno K, Ushiki J and Higo K: Involvement of the active metabolites in the inhibitory activity of K579 on rat plasma dipeptidyl peptidase IV. Eur J Pharmacol 505: 237-241, 2004.

11. Takasaki K, Nakajima T, Ueno K, Nomoto Y and Higo K: Effects of combination treatment with dipeptidyl peptidase IV inhibitor and sulfonylurea on glucose levels in rats. J Pharmacol Sci 95: 291-293, 2004.

12. Dubé PE and Brubaker PL: Frontiers in glucagon-like peptide-2: Multiple actions, multiple mediators. Am J Physiol Endocrinol Metab 293: E460-E465, 2007.

13. Drozdowski L and Thomson AB: Intestinal hormones and growth factors: Effects on the small intestine. World J Gastroenterol 15 : 385-406, 2009

14. Hartmann B, Thulesen J, Kissow H, Thulesen S, Orskov C, Ropke C, Poulsen SS and Holst JJ: Dipeptidyl peptidase IV inhibition enhances the intestinotrophic effect of glucagon-like peptide-2 in rats and mice. Endocrinology 141: 4013-4020, 2000.

15. Boushey RP, Yusta B and Drucker DJ: Glucagon-like peptide 2 decreases mortality and reduces the severity of indomethacininduced murine enteritis. Am J Physiol 277: E937-E947, 1999.

16. Fujiwara $\mathrm{K}$, Inoue $\mathrm{T}$, Yorifuji $\mathrm{N}$, Iguchi $\mathrm{M}$, Sakanaka $\mathrm{T}$, Narabayashi K, Kakimoto K, Nouda S, Okada T, Ishida K, et al: Combined treatment with dipeptidyl peptidase 4 (DPP4) inhibitor sitagliptin and elemental diets reduced indomethacin-induced intestinal injury in rats via the increase of mucosal glucagonlike peptide-2 concentration. J Clin Biochem Nutr 56: 155-162, 2015 .
17. Inoue T, Higashiyama M, Kaji I, Rudenkyy S, Higuchi K, Guth PH, Engel E, Kaunitz JD and Akiba Y: Dipeptidyl peptidase IV inhibition prevents the formation and promotes the healing of indomethacin-induced intestinal ulcers in rats. Dig Dis Sci 59: 1286-1295, 2014.

18. Iakoubov R, Lauffer LM, Trivedi S, Kim YI and Brubaker PL: Carcinogenic effects of exogenous and endogenous glucagon-like peptide-2 in azoxymethane-treated mice. Endocrinology 150: 4033-4043, 2009.

19. Yu DM, Yao TW, Chowdhury S, Nadvi NA, Osborne B, Church WB, McCaughan GW and Gorrell MD: The dipeptidyl peptidase IV family in cancer and cell biology. FEBS J 277: 1126-1144, 2010

20. Inoue T, Murano M, Yoda Y, Kuramoto T, Kakimoto K, Ishida K, Kawakami K, Abe Y, Morita E, Murano N, et al: R-etodolac induces E-cadherin and suppresses colitis-related mouse colon tumorigenesis. Oncol Rep 24: 1487-1492, 2010.

21. Femia AP, Raimondi L, Maglieri G, Lodovici M, Mannucci E and Caderni G: Long-term treatment with Sitagliptin, a dipeptidyl peptidase- 4 inhibitor, reduces colon carcinogenesis and reactive oxygen species in 1,2-dimethylhydrazine-induced rats. Int J Cancer 133: 2498-2503, 2013.

22. Fisher M and Knappertz V: The dose of aspirin for the prevention of cardiovascular and cerebrovascular events. Curr Med Res Opin 22: 1239-1248, 2006.

23. Berger JS, Brown DL and Becker RC: Low-dose aspirin in patients with stable cardiovascular disease: A meta-analysis. Am J Med 121: 43-49, 2008

24. Higuchi K, Umegaki E, Watanabe T, Yoda Y, Morita E, Murano M, Tokioka S and Arakawa T: Present status and strategy of NSAIDs-induced small bowel injury. J Gastroenterol 44: 879-888, 2009.

25. Ito Y, Sasaki M, Funaki Y, Ogasawara N, Mizuno M, Iida A, Izawa S, Masui R, Kondo Y, Tamura Y, et al: Nonsteroidal antiinflammatory drug-induced visible and invisible small intestinal injury. J Clin Biochem Nutr 53: 55-59, 2013.

26. Kannen V, Garcia SB, Stopper H and Waaga-Gasser AM: Glucagon-like peptide 2 in colon carcinogenesis: Possible target for anti-cancer therapy? Pharmacol Ther 139: 87-94, 2013.

27. Sakanaka T, Inoue T, Yorifuji N, Iguchi M, Fujiwara K, Narabayashi K, Kakimoto K, Nouda S, Okada T, Kuramoto T, et al: The effects of a TGR 5 agonist and a dipeptidyl peptidase IV inhibitor on dextran sulfate sodium-induced colitis in mice. J Gastroenterol Hepatol 30 (Suppl 1): S60-S65, 2015.

28. Fujiwara K, Inoue T, Yorifuji N, Iguchi M, Okada T, Kakimoto K, Nouda S, Kawakami K, Abe Y, Takeuchi T, et al: Su2022 Sitagliptin, a dipeptidyl peptidase-4 inhibitor, suppresses CXCL5 and Sdf-1 and does not accelerate intestinal neoplasia formation in $\mathrm{APC}^{\mathrm{MIN} /+}$ mice fed a high fat diet. Gastroenterology 148 (Suppl 1): S-577, 2015.

29. Lin CT, Tang HY, Han YS, Liu HP, Huang SF, Chien CH, Shyy J, Chiu JJ and Chen X: Downregulation of Signaling-active IGF-1 by Dipeptidyl Peptidase IV (DPP-IV). Int J Biomed Sci 6: 301-309, 2010.

30. Ikeda T, Kumagai E, Iwata S and Yamakawa A: Soluble CD26/ Dipeptidyl Peptidase IV Enhances the Transcription of IL-6 and TNF- $\alpha$ in THP-1 Cells and Monocytes. PLoS One 8: e66520, 2013.

31. Reinhold D, Bank U, Bühling F, Neubert K, Mattern T, Ulmer AJ, Flad HD and Ansorge S: Dipeptidyl peptidase IV (CD26) on human lymphocytes. Synthetic inhibitors of and antibodies against dipeptidyl peptidase IV suppress the proliferation of pokeweed mitogen-stimulated peripheral blood mononuclear cells, and IL-2 and IL-6 production. Immunobiology 188: 403-414, 1993. 